East African Medical Journal Vol. 78 No. 7 July 2001

FREQUENCY OF ADENOTONSILLECTOMY IN SOME NAIROBI HOSPITALS

H.O. Oburra, MMed, FRCSE, Senior Lecturer, Department of Surgery, College of Health Sciences, University of Nairobi, P.O. Box 19676, Nairobi and M. Idenya, MbChB, Resident, Department of ENT/HN Surgery, Kenyatta National Hospital, P.O. Box 20728, Nairobi.

Request for reprints to: Dr. H. O. Oburra, Department of Surgery, Coltege of Health Sciences. University of Nairobi, P.O. Box 19676 Nairobi.

\title{
FREQUENCY OF ADENOTONSILLECTOMY IN SOME NAIROBI HOSPITALS
}

\author{
H. O. OBURRA and M. IDENYA
}

\begin{abstract}
Objectives: To determine the frequency of adenotonsillectomy in a sample of Kenyan hospitals and to review indications, timing and complications in 97 cases of adenotonsillectomy done by the authors.

Design: Retrospective, descriptive study.

Setting: Kenyatta National, Nairobi, Aga Khan, Gertrude and Mater Hospitals.

Results: Adenotonsillectomy is the most frequent otolaryngologic surgical operation. Indications for surgery were upper airway obstruction in $61.3 \%$, recurrent tonsillitis in $28.7 \%$ and both in $7.5 \%$. Surgery was indicated during the acute stage in $6.8 \%$ of cases. There was one case of post-operative acute airway obstruction. Post operative bleeding from the tonsillar bed was encountered in $2.1 \%$ of cases.

Conclusion: Adenotonsillectomy is the most common otolaryngologic surgical operation in our set-up. The low frequency of complications and a short hospital stay puts up a case for routine adenotonsillectomy as a day surgery procedure.
\end{abstract}

\section{INTRODUCTION}

Adenotonsillectomy is one of the most common elective otolaryngologic operations in Kenyan Ear Nose and Throat/Head and Neck (ENT/HN) surgical units. This is in contrast to the situation in the United Kingdom where the operation is on the decline(1). By convention the operations done on tonsils and adenoids are carried out as elective procedures(2). The main indications for removal of these lymphoid tissues of the Waldeyer's ring are upper airway obstruction and recurrent infection compromising regular activities of an individual. Other less frequent indications are quinsy (peritonsillar abscess), for access to the structures behind the tonsillar bed, during pharyngeal reconstruction to alleviate snoring, as a part of anti tumour surgery for neoplams in the vicinity and infestation of the tonsil by tonsilloliths causing halitosis. Tonsillectomy following quinsy or peritonsillar abscess is usually carried out as an elective procedure, weeks after primary drainage of the abscess. Few surgeons would advocate tonsillectomy during the acute inflammatory period for fear of haemorrhage, difficulties in dissection and spread of infection. It is however becoming increasingly apparent that adenotonsillectomy may be indicated in the acute inflammatory period in well defined situations especially in acute airway obstruction in inflamed hypertrophied tonsils which may also occur in immunesuppressed states. Adenotonsillectomy has become a very safe procedure following the perfection of the technique and anticipatory precautions against foreseen complications. Post tonsillectomy haemorrhage, which was formally well documented, is a relatively uncommon occurrence in ENT/HN units.
In this study, the frequency of adenotonsillectomy is determined from the operation registers maintained in five main city hospitals and a retrospective review of cases undergoing in-patient adenoidectomy, tonsillectomy or adenotonsillectomy done by the authors in various Nairobi hospitals, is done to elicit the current indications, complications and technique of the operation. Summary case notes of two patients one having tonsillectomy during the acute inflammatory stage and another one having tonsillitis complicated by epiglottitis are also presented. For purposes of this study, the term adenotonsillectomy is used to define the removal of adenoids, palatine tonsils or both.

\section{MATERIALS AND METHODS}

Operating theatre registers at Kenyatta National Hospital. Aga Khan and Gertrude Garden Children's Hospital were analysed to establish the frequency of adenotonsillectomy. Case notes of consecutive admissions for tonsillectomy, adenoidectomy or adenotonsillectomy by the authors at the Aga Khan, The Nairobi and Gertrude Garden Children's Hospitals between 1992 and 1999 were scrutinised for indications, timing and technique of the operation and post operative complications.

\section{RESULTS}

Frequency of adenotonsillectomy: Adenotonsillectomy was the most frequent ENT/HN operation in all the three city hospitals. Out of 1148 ENT/HN surgical operations done in 1999 at the $\mathrm{KNH}$ operating theatres, $251(21.8 \%)$ were adenotonsillectomy while in Aga Khan Hospital, out of $404 \mathrm{ENT} / \mathrm{HN}$ surgical operations done in 1998 and 1999, 131 (48.2\%) were adenotonsillectomies. 
In Gertrude Gardens Children Hospital, the corresponding figures were 276 total ENT/HN surgical operations of which $206(74.6 \%)$ involved adenotonsillectomy, thus emphasising an even higher frequency of the operation in paediatric otolaryngology.

Indications for adenotonsillectomy: Ninety seven patients were admitted for adenoidectomy, tonsillectomy or adenotonsillectomy by the authors. The indications were as shown in Table 1. Figure I shows the age prevalence of upper airway obstruction and recurrent tonsillitis as indications for tonsillectomy in 89 patients. The indications for surgery were upper airway obstruction in $58.8 \%$, recurrent tonsillitis in $23.7 \%$, both upper airway obstruction and recurrent tonsillitis in $10.7 \%$ and quinsy in $5.3 \%$. Six surgical operations were done during the acute stage. Two of these were adults with unrelenting symptoms of quinsy after two days of trial antibiotics and no evidence of pus on aspiration, two were children with rapidly progressive respiratory failure and exhaustion from sleep apnoea and two had acute obstructive symptoms from hypertrophied acute tonsillitis needing emergency intubation and ICU admission. Four adult and one paediatric patient with sleep apnoea syndrome had preoperative diagnosis of immunesupression from HIV infection.

Table 1

Indications for adenotonsillectomy

\begin{tabular}{lcc}
\multicolumn{3}{c}{ Indications for adenotonsillectomy } \\
\hline Indication & No. & $\%$ \\
\hline & 57 & 58.8 \\
Upper airway obstruction & 23 & 23.7 \\
Recurrent tonsillitis & & \\
Upper airway obstruction & 10 & 10.3 \\
and recurrent tonsillitis & 5 & 5.2 \\
Quinsy & 2 & 2.1 \\
Tonsilloliths & \\
\hline
\end{tabular}

Figure 1

Age prevalence of adentonsillectomies indications.s

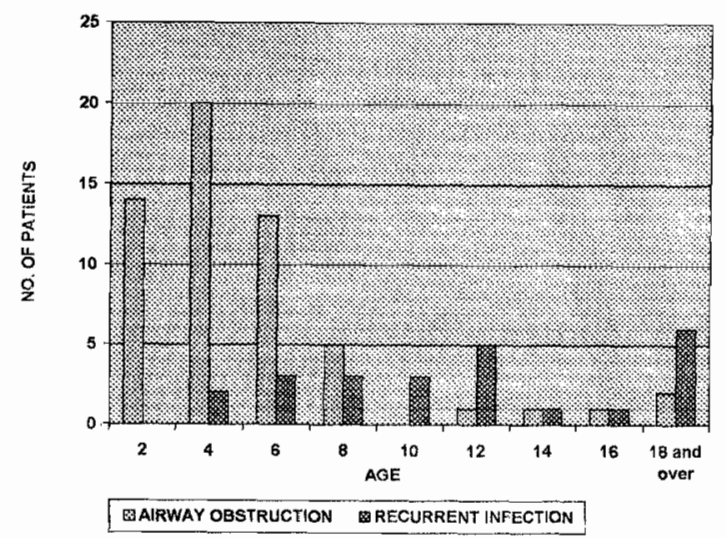

Complications of adenotonsillectomy: The standard operation technique was tonsillectomy by dissection technique and adenoidectomy using adenoid curettes and haemostasis being achieved by coagulation, the operation being done under general anaesthesia. One patient developed acute airway obstruction from oedematous uvula six hours post operatively needing emergency intubation and uvulectomy. Two patients had postnasal pack inserted for 24 hours to control persistent bleeding from the adenoid bed. One patient had primary postoperative haemorrhage from the tonsillar bed, which resolved with conservative measures and another one developed secondary haemorrhage at day seven post operatively.

\section{Table 2}

Complications of adenotonsillectomy

\begin{tabular}{|c|c|c|c|}
\hline Complication & & No. & $\%$ \\
\hline Haemorrhage & & 2 & 2.1 \\
\hline Primary haemorrhage & 1 & & \\
\hline Secondary infection & 1 & & \\
\hline Failure to improve airway: & & 12 & 12.4 \\
\hline Total failure & 3 & & \\
\hline Persistent significant upper & & & \\
\hline airway obstruction & 9 & & \\
\hline Delayed onset of feeding on solids & & 8 & 8.2 \\
\hline Upper respiratory tract infection & & 2 & 2.1 \\
\hline Post operative somnolence & & I & 1.0 \\
\hline Prolonged healing of the tonsillar bed & & 1 & 1.0 \\
\hline Anaesthetic complications: & & 3 & 3.1 \\
\hline $\begin{array}{l}\text { kinked ET tube with severe } \\
\text { bradycardia } \\
\text { accidental premature post operation }\end{array}$ & 1 & & \\
\hline extubation with cerebral damage & i & & \\
\hline $\begin{array}{l}\text { Bleeding from nasal turbinate } \\
\text { traumatised by nasotracheal tube }\end{array}$ & 1 & & \\
\hline No complicat1ons & & 68 & 70.1 \\
\hline Total & & 97 & 100.0 \\
\hline
\end{tabular}

Delayed onset in feeding prolonging postoperative stay was seen in eight patients who could only tolerate fluids for a period ranging from three to twenty seven days. The rest of the patients were on oral solid fecds within 48 hours. Three ol these patients were 15 years old. Severe anaesthetic complications were seen in two patients who had severe bradycardia following ET tube kinking and occlusion at surgery and another one who had accidental self-extubation developing cerebral ischaemia and permanent brain damage. The latter patient had had sleep apnoea since neonatal period. Apnoea probably due to lost $\mathrm{CO}_{2}$ dependent respiratory drive developed immediately after accidental self-extubation causing severe cerebral ischaemia before re-establishment of the airway. Thus a total of $29.9 \%$ of cases had complications (Table 2). However, only five patients $(5.2 \%)$ had complications that would have necessitated re-admission or retention in the hospital had the operation been done on an outpatient basis.

Presented below are summary case notes of two patients with complications of tonsillectomy needing 
emergency endotracheal intubation, one undergoing adenotonsillectomy during the acute inflammatory stage.

Case 1: MM, a three and a half-year old male child presented to the Accident and Emergency Unit of Nairobi Hospital with acute upper airway obstruction following a two-day history of sore throat. On examination epiglottitis was suspected, he was admitted, intubated and transferred to ICU. At intubation, he was noted to have septic hypertrophied tonsils obstructing oropharynx. Adenotonsillectomy was done and the child extubated in the immediate postoperative period. Total blood count showed a leucocytosis of 12,000. Blood biochemistry, and serology were normal. Superficial tonsillar cultures revealed beta haemolytic streptococci.

Case 2: A three-year old child presented to the Emergency unit with gradual onset upper airway obstruction. He had been earlier diagnosed by an ENT/HN surgeon as a case of recurrent tonsillitis and scheduled for elective adenotonsillectomy. At the emergency unit he was noticed to prefer sitting upright and in obvious respiratory distress. He was not hoarse. After emergency endotracheal intubation he was noticed to have follicular tonsillitis below which were swollen epiglottis compromising the supraglottic airway. He was started on intravenous amoxicillin/clavulinic acid injections and successfully extubated on the third day and discharged on oral medications on the fourth day and underwent an elective adenotonsillectomy four weeks later. Tonsillar swab cultures grew beta haemolytic streptococci. In case 2 , co-existent epiglottis ruled out immediate benefit from emergency tonsillectomy.

\section{DISCUSSION}

Palatine, lingual and nasopharyngeal tonsils are immunereactive lymphoid tissue located around the oropharynx in an arrangement commonly referred to as the Waldeyer's ring. Similar Iymphoid tissue are located in the posterior pharyngeal wall and the intestinal Peyer's patches. The nasopharyngeal tonsil which is located behind the nasal cavity is better known as adenoids while the palatine tonsils are usually referred to as the 'tonsils' by convention. Inflammation or hypertrophy of these two structures causes the majority of symptoms from these lymphoid tissues and hence adenotonsillectomy refers to surgical removal of these two latter lymphoid structures.

The prominence of operations on the tonsils and adenoids as the most common elective ENT/HN operation in Kenyan hospitals is expected. Gertrude Gardens Children's Hospital figures attest to this. However the hospital figures at the $\mathrm{KNH}$ present a more balanced overview of the scope of ENT/HN operations in Kenya because it is the only fully fledged unit in the country. The high frequency of inadequately treated upper respiratory tract infections and the depressed immunological status in our socioeconomic environment could be responsible. This is in contradiction to the situation in North America and Europe where myringotomy and insertion of grommets for otitis media with effusion is the most frequently performed otolaryngologic surgery $(3,4)$ while the frequency of adenotonsillectomy has tended to decline(1).

The operation of tonsillectomy has been plagued with controversies over the years partly because of failure of otolaryngologists and paediatricians to appreciate the central role of the Waldeyer's ring lymphoid tissue in immunological development of a child but also because of the difficulties in formulating a precise definition of adenotonsillitis. However, during the last three decades, conservatism and strict selection criteria has reduced liberal removal of the tonsils and adenoids for infection thus leaving obstructive syndromes with sleep apnoea as the most frequent indication for adenotonsillectomy.

Although the initial infection could be viral, most frequent causative organisms in adenotonsillitis are streptococcus pneumoniae, Staphylococcus aureus, Haemophilus influenzae and beta haemolytic streptococci(5). Organisms causing tonsillitis are, however, difficult to asses by simple throat swabs as the same organisms can be cultured in routine swabs from normal throats. Moreover, cultures from the centre of a tonsillectomy tissue specimen are frequently different from tonsillar surface swabs.

The most common indications for adenotonsillectomy are upper airway obstruction and recurrent infective inflammation. The clinician must appreciate that the size of the tonsils and adenoids and the frequency of tonsillitis are variable and depends on age, familial factors, exposure to infection and immunological activity. It must also be realised that the immunological activity is not necessarily immunecompetent activity. Consequently very large or frequently infected tonsils in our set up in the relevant age group may sometimes indicate immunesuppression or delay in development of fractions of the immunoglobulins (such as IgGl, IgG2 or IgG3), congenital $\mathrm{T}$ cell or phagocytosis defects. In such circumstances, there may be associated upper airway infections such as sinusitis or recurrent pneumonia. It may also indicate Type One hypersensitivity. These are the fine facts that one has to consider before contemplating removal of the Waldeyer ring lymphoid tissue for recurrent infection.

Upper airway obstructive phenomenon causing sleep apnoea syndrome and its associated cardiopulmonary, gastrointestinal, and mandibulofacial complications and even growth retardation have been exhaustively reviewed in literature (6-8).

While the size of tonsils can be easily assessed by visual inspection, the adenoid size can only be inferred from lateral soft tissue nasopharyngeal plain radiographs. However, radiological appearance alone cannot be an indication for adenotonsillectomy. The operation of elective adenotonsillectomy to alleviate upper airway obstruction must be weighed against the physiological role of these lymphoid tissues particularly in the young child and the fact that the younger the child, the less dramatic the alleviation of the symptoms. The latter finding is because of the ease with which adenoid tissue grows to re-occupy 
the still small capacity nasopharyngeal space thus necessitating repeat surgery. In circumstances where there is impeding respiratory failure from prolonged sleep apnoea with lack of REM sleep, the alleviation of the obstruction with initial endotracheal intubation and emergency adenotonsillectomy is warranted.

Recurrent tonsillitis as an indication for tonsillectomy is frequently based on subjective premises and hence is open to abuse. Recent attempts to define tonsillitis in gross clinical terms including generalised erythematous reaction, pus or yellow exudate from the crypts, inflammatory membrane or ulceration on the surface of the tonsils and cervical lymphadenopathy is far from perfect(2). Paediatricians and otolarynglogists still lack common grounds and foolproof criteria for defining tonsillitis. Paradise et al(9) showed the fallacy of relying on vague symptoms such as sore throat to define adenotonsillitis. Moreover in certain cases of chronic or recurrent tonsillitis, the tonsils may appear small and fibrotic and pus may only be encountered during surgical manipulation. The famous guideline of "six attacks of tonsillitis per year for two years' is just a rough guideline.

Tonsils and adenoids are frequently involved in immunesuppresion due to HIV infection. The recurrent tonsillitis and obstructive enlargement are a common occurrence. In the former state, the inflammatory process frequently spills over to the anterior pillar and uvula. But perhaps, the most grave effect of adenotonsillar hypertrophy in an AIDS situation is sleep apnoea and exhaustion of a patient whose reserves for stress reaction are already compromised. When performing adenotonsillectomy in patients with immunesuppression secondary to HIV infection, close attention must be directed to the patient's general condition, coagulation status, the situation of the healing processes, the stage of the illness using the CD4 counts, the viral load and the presence of serious opportunistic infections.

Adenotonsillectomy during the acute inflammatory illness is usually avoided by many surgeons for fear of excessive intraoperative bleeding, provocation of local spread of infection or bacteraemia and accentuation of postoperative morbidity. These factors are more theoretical than born in practice(10). First of all, the vast majority of surgeons perform tonsillectomy under antibiotic cover even during an elective operation, and in quinsy, patients almost invariably have been on high doses of antibiotics without any signs of relief. Secondly, tonsillectomy during an acute period such as that encountered in quinsy or HIV infection is an entirely different technique that has to be mastered. Of foremost importance, the friable tonsil must not be grasped with a tonsil holding forceps. Instead, a plane of cleavage is developed and the tonsil is simply peeled off the tonsillar bed using a blunt instrument such as tonsil dissector or even a Yankaur's suction nozzle. In the acutely inflamed tonsil, the plane of cleavage is well defined and the much written about haemorrhage is paradoxically very minimal. Contrary to popular belief, dangers associated with acute rupture of a peritonsillar abscess and inhalation of the infected material either at normal sleep or during endotracheal intubation is rarely, if ever, observed in clinical practice although rapid upper airway compromise can rapidly occur in quinsy.

In managing quinsy, the clinician must appreciate that the term quinsy does not necessarily imply a peritonsillar abscess, as negative taps are very frequent in such patients. The most important problem that drives a quinsy patient to seck relief from a physician is the morbidity associated with it including pain, total dysphagia, drooling and obstructive sleep apnoea. It is for alleviation of these symptoms that various modes of management including incision and drainage followed by staged tonsillectomy, simple aspiration, intravenous antibiotics and 'abscess tonsillectomy' have been advocated by many authors(11). In practice, however, many paticnts still spend long duration of time with this morbidity on trial antibiotics while others undergo fruitless negative incisions because of the paucity of reliable signs for the presence of a ready abscess cavity. The two points against 'abscess' tonsillectomy is the fact that most cases of quinsy are not related to recurrent tonsillitis and that recurrent quinsy claimed in literature is not a universal finding.

In the current status of medical knowledge, developments in microbiologic control and ready availability for training of otolaryngologists, precise indications exist for tonsillectomy during the acute inflammatory stage. And one such indication is the quinsy sufferer on intravenous augmented penicillin or any other corresponding broad spectrum antibiotic who show no alleviation of pain, resolution of the tonsillar swelling or settling of raised temperatures and who have no signs of ready abscess. Such a patient should have trial aspiration and incission and drainage preferably under local anaesthesia. Failure to elicit pus should warrant removal of the tonsils at the same sitting. Other such indications include are acute airway obstruction from inflamed hypertrophied tonsils commonly found in immunesuppressed states.

Removal of tonsils and adenoids to control gluc ear remains controversial. Many studies have shown contradictory results $(12,13)$ and it is admittedly difficult to design such a study in a situation where many variables, some unknown, exist. Currently, recurrent acute or serous otitis media alone are not indications for adenotonsillectomy unless recognised indications likc sleep apnoea or frequent tonsillitis exist. Removal of tonsils for the sole purpose of preventing sickle cell crisis, rheumatic fever, glomerulonephritis or other immunological illnesses in none predisposed individuals has not been objectively shown to be beneficial.

Complications following adenotonsillectomy have diminished following restriction of the operation to suitably trained otolaryngologists. The once frequent cases of post-operative haemorrhage have considerably diminished. A review by Choudhury et al(14) found an incidence of post adenotonsillectomy haemorrhage of $2.5 \%$. In this series, there was a $2.1 \%$ frequency of post operative 
bleeding and a nasopharyngeal pack was left in situ in a further two patients. Despite global reduction of the frequency of post-operative haemorrhage, measures to prevent blood loss need to be taken even more seriously in the Kenyan scenario in view of the high prevalence of HIV infection which make blood transfusions hazardous. Studies to asses the frequency of other complications like rhinolalia aperta, eustachian tube damage and the rare dislocation of the atlanto-occipital joint are lacking in literature. Kinking of the endotracheal tube and the rare accidental self extubation resulting in cerebral ischaemic damage are reminders of the hazards that stalk otolaryngologists whose nature of operations force them to encroach on the anaesthetists field of operations. Currently, special angulated tubes have been designed to reduce episodes of kinking and occlusion of the tube during surgical operation. It is worth noting that only $5.2 \%$ of patients would have been retained or re-admitted in the hospital for observation or active management of a complication. This makes tonsillectomy a reasonably safe day surgery procedure.

However, not all surgical operations are successful. It is worth noting that adenotonsillectomy in three patients with upper airway obstruction resulted in total failure to improve the airway while nine other patients had some significant obstruction post operatively. In the enthusiasm to operate, one should not forget that other important processes like rhinitis and general airway oedema or even choanal stenosis may be contributors to the upper airway blockage and that what may appear to be recurrent tonsillitis may indeed be a pharyngeal manifestation of a systemic problem.

\section{REFERENCES}

1. Black, N.A. Geographical variations in the use of surgery for glue ear. J. Roy. Soc. Med. 1985; 78:641-648.

2. Kerr A.G. Scott-Brown's Otolaryngology vol. 6. 6th Edition. 1997. Ch18. Edited by Cowan D.I. and Hibbert J. Hienman Butterworth

3. Rasmussen F. Protracted secretory otitis media. The impact of familial factors and day care centre attendance. Intern. J. Patdiat. Otol. 1993; 26: 29-37.

4. Schilder A.G.M., Zielhuis G.A. and Van Den Broek P. The otological profiles of a cohort of Dutch 7.5 to 8 year olds. Clin. Otol. 1993; 18: 48-54.

5. Box O.T., Cleveland R.T. and Willard C.Y. Bacterial flora of the upper respiratory tract. Amer. J. Dis. Child. 1961; 102: 293-330

6. Mangat D., Orr C. and Smith R.C. Sleep apnoea, hypersomnolence and upper airway obstruction secondary to adenotonsillar enlargement. Arch. Otol. 1977; 103: 383-386.

7. Eliaschar I., Lavie P. and Halperin E. et al. Sleep apnoeic episodes as indications for adenotonsillectomy. Arch. Otol. 1980; 106: 492-496.

8. Mauer K.W.. Staats B.A. and Olsen K.D. Upper airway obstruction and disordered nocturnal breathing in children. Mayo Clinic Proceedings, 1983; 58: 349-353.

9. Paradise J.L., Bluestone C.D. and Bachman R.Z. et al. History of recurrent sore throats as an indication for tonsillectomy. N. Engl. J. Med. 1978; 298: 409-413.

10. Moesgaard Niebont and Gricsson I. Peritonsillar abscess. Cases treated with tonsillectomy a chaud. J. Laryng. Otol. 1981;95:801-807

11. Snow D.L., Campbell J.B. and Morgan D.W. Management of peritonsillar sepsis by needle aspiration. Clinical Otol. 1991; 16: 245-247.

12. Bulman C.H., Brook, S.J and Berry M.G. A prospective randomised trial of adenoidectomy vs. grommet insertion in the treatment of ghe ear. Clin. Otol. 1984: 9: 67-75.

13. Widemar L., Svenson C., Rynnel-Dagloo B. and Schiratzki H. The effect of adenoidectomy on secretory otitis media; a two year controlled prospective study. Clin. Otol. 1985; 10: 345-350.

14. Choudhury K., Tewfik T.K. and Schloss M.D. Post tonsillectomy and adenoidectomy haemorrhage. J. Otol. 1988; 17: 46-49. 